\title{
DISTANCIAMENTO SOCIAL DO IDOSO SAUDÁVEL DURANTE A PANDEMIA COVID-19: POSSIBILIDADES E DESAFIOS
}

\author{
Carla Argenta' \\ ORCID: 0000-0002-9729-410 \\ Daniella Pires Nunes" \\ ORCID: 0000-0002-4679-0373
}

Karina Silveira de Almeida Hammerschmidt"II ORCID: 0000-0002-7140-3427

Luciana Mitsue Sakano Niwa'v ORCID: 0000-0002-9342-7454

Priscilla Alfradique de Souzav ORCID: 0000-0002-4625-7552

Priscila de Oliveira Cabral Melovi ORCID: 0000-0002-6105-2248

'Universidade do Estado de Santa Catarina. Chapecó, Santa Catarina, Brasil.

"Universidade Estadual de Campinas. Campinas, São Paulo, Brasil.

"' Universidade Federal do Paraná. Curitiba, Paraná, Brasil.

"'Universidade de São Paulo. São Paulo, São Paulo, Brasil.

v Universidade Federal do Estado do Rio de Janeiro. Rio de Janeiro, Rio de Janeiro, Brasil.

v'Universidade Federal de Alagoas. Maceió, Alagoas, Brasil

Autor Correspondente: Carla Argenta E-mail: carla.argenta@udesc.br

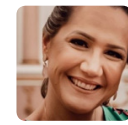

Como citar: Argenta C, Nunes DP, Hammerschmidt KSA, Niwa LMS, Souza PA, Melo POC. Distanciamento social do idoso saudável durante a pandemia covid-19: possibilidades e desafios. In: Santana RF. Enfermagem gerontologica no cuidado do idoso em tempos da COVID 19. 2.ed.rev. Brasilia, DF: Editora ABEn; 2020. p.5-10. (Serie Enfermagem e Pandemias, 1). https://doi.org/10.51234/aben.20.e01.c01

\section{INTRODUÇÃO}

Os coronavírus humanos (HCoVs) são considerados vírus respiratórios comumente associados com doenças do trato respiratório, que variam em termos de gravidade, podendo inclusive ser fatal. Há sete tipos de coronavírus humanos conhecidos, dentre eles o SARS-CoV-1 (que causa síndrome respiratória aguda grave) e o SARS-CoV-2 (vírus que causa a doença COVID-19)' ${ }^{1}$.

O primeiro caso do novo coronavírus (SARS-CoV-2) foi notificado em Wuhan, China, em dezembro de 2019. O aumento significativo no número de casos, bem como das mortes e dos países afetados, motivou em 30 de janeiro de 2020, a Organização Mundial da Saúde (OMS) declarar Emergência de Saúde Pública Internacional e em 11 março de 2020, foi assumido como pandemia ${ }^{2}$ No Brasil, o primeiro caso positivo foi anunciado em 26 de fevereiro de 2020 e o primeiro óbito em 17 de março de $2020^{3}$, sendo declarado Emergência de Saúde Pública Nacional em 3 de fevereiro de $2020^{4}$.

A presença do vírus circulante e ausência de imunidade prévia na população humana, aliada a inexistência de vacina contra SARS-CoV-2, fez com que o número de casos aumentasse exponencialmente ${ }^{5}$. O risco de morrer por COVID-19 aumenta com a idade, principalmente para os idosos com doença crônica, com destaque para aqueles com hipertensão arterial e diabetes mellitus ${ }^{6}$. A prevalência maior em idosos se dá devido a imunossenescência ${ }^{7}$, que induz a diminuição da eficácia do sistema imunológico ${ }^{5}$, caracterizando essas pessoas como uma população de risco. Reforça esta preocupação os dados epidemiológicos que apontam incidência de mortalidade em 14,8\% dos idosos com 80 anos ou mais infectados, $8 \%$ de 70 a 79 anos e $8,8 \%$ dos idosos com 60 a 69 anos $^{8}$, intensificando as apreensões com essa população.

Dessa forma, diversos esforços foram desenvolvidos pelas organizações de saúde e poder público para conter o 
avanço da disseminação do SARS-CoV-2. Dentre os planos de contingência de diversos países, destacaram-se medidas para limitar a propagação do vírus no âmbito local, nacional e internacional, como: distanciamento social, triagem dos doentes suspeitos, restrições de viagens, vigilância, quarentena dos casos suspeitos, antecipação de vacinação da Influenza, suspensão de aulas e atividades comerciais e laborais ${ }^{9}$.

O distanciamento social, de modo especial, para as populações de risco, trata-se de tentativa para proteger os vulneráveis (em relação a contaminação pelo SARS-CoV-2), envolvendo idosos saudáveis, que devem permanecer em seus domicílios, porém com preservação da autonomia e independência. No entanto, é sabido que o distanciamento social, como medida preventiva, pode desencadear problemas de ordem fisiológica, psicológica e social neste momento pandêmico.

Diante da importância e necessidade do distanciamento social versus saúde multidimensional do idoso, emergiu o desejo de refletir sobre o assunto, à luz da literatura atual, tecendo considerações sobre as possibilidades e desafios a serem enfrentados junto aos idosos saudáveis, famílias e enfermagem gerontológica.

\section{OBJETIVO}

Refletir sobre o distanciamento social do idoso saudável provocado pela pandemia COVID-19, tecendo considerações a respeito dos desafios e possibilidades para o idoso, a família e a enfermagem gerontológica.

\section{MÉTODO}

Trata-se de texto teórico-reflexivo que versa sobre os desafios e possibilidades, oriundos do distanciamento social, para o idoso e seus familiares durante a pandemia da COVID-19, assim como para a enfermagem gerontológica.

As reflexões foram embasadas em artigos internacionais e nacionais relacionados ao tema, bem como nas vivências das autoras. Assim, as considerações foram categorizadas em três grandes pilares: 1) repercussões da pandemia para o idoso, incluindo a vulnerabilidade deste; 2 ) desafios que o distanciamento social apresenta para os idosos saudáveis; 3 ) possibilidades de ações para os idosos saudáveis (sem diagnóstico de COVID-19) em distanciamento social, envolvendo a família e a enfermagem gerontológica.

\section{IDOSO COMO GRUPO DE RISCO PARA SARS-COV-2}

A população idosa apresenta maior vulnerabilidade ao coronavírus. No Brasil, até o dia 16 de abril de 2020 foram registrados 1924 óbitos por COVID-19. Destes, $72 \%$ ocorreram em pessoas de $60+$ anos ${ }^{4}$. O processo fisiológico do envelhecimento incita a diminuição da eficácia do sistema imune, aumentando propensão a morbidade e mortalidade de doenças infecciosas. As alterações da imunossenescência ${ }^{7}$ associadas a redução da expansibilidade torácica, da eficiência das trocas gasosas e da força dos músculos respiratórios ${ }^{10}$, tornam os idosos mais suscetíveis e com mau prognóstico ao COVID-19. Além disso, idosos com comorbidades como cardiopatia, diabete mellitus e doenças pulmonares têm maior chance de desenvolver graves e críticas condições da doença e isso implica em diversas internações nas unidades de terapia intensiva e alta taxa de mortalidade ${ }^{11}$.

A imunossenescência é processo natural do envelhecimento, que aumenta de modo geral, a incidência de doenças infectocontagiosas em idosos como gripe, resfriados comuns e COVID-19. Deste modo, por exemplo, as pessoas idosas residentes em Instituições de Longa Permanência são consideradas de alto risco para a infecção pelo SARS-CoV-2 por, geralmente, apresentarem: doenças crônicas, idade avançada, dependência, convivência em aglomerados e contato frequente com profissionais ${ }^{12}$.

Sendo assim se destaca que alguns municípios têm focado em ações de estratificação de risco e ressaltam que tem sido fundamental para o enfrentamento da COVID-19, sendo planejada não apenas de acordo com a idade do idoso, mas sim considerando o índice de vulnerabilidade clínico-funcional. Sendo assim, com 
base no risco de complicações que a COVID-19 representa para os idosos, particularmente os idosos frágeis, estes devem ser monitorados rigorosamente pela atenção primária à saúde ${ }^{13}$.

\section{DESAFIOS DO DISTANCIAMENTO SOCIAL PARA IDOSOS E FAMILIARES}

A participação social e a integração dos idosos são aspectos importantes para o envelhecimento saudável. Dentre os desafios que envolvem este envelhecimento, está a solidão do idoso, definida como reação emocional de insatisfação provocada pela ausência ou deficiência de relacionamentos significativos, que inclui algum tipo de isolamento; trata-se de sentimento penoso e doloroso, de carência que faz referência aos outros. Nesse público, a solidão pode propiciar declínio da saúde mental e sintomas indicativos de depressão, consumo de substâncias ilícitas e tentativas de suicídio ${ }^{14}$.

Além disso, nesse período pandêmico, a solidão pode aumentar o desamparo do idoso, com percepção de exclusão da família e da sociedade, sobretudo devido à exibição vivida mediante afloramento das atitudes de ageísmo. No entanto, reforça-se que o distanciamento social não significa abandono ou desamparo, ao contrário, acredita-se que esse pode ser momento em que o idoso necessita com maior ênfase da rede de apoio para sentir-se protegido e acolhido.

O abandono, o desrespeito e a amplitude de tempo no domicílio podem incitar a violência contra o idoso, praticada por familiares, cuidadores e/ou desconhecidos. Desse modo, atenção especial deve voltar-se aos idosos durante o distanciamento social, principalmente para os que possuem fatores de risco à violência, que incluem aqueles com: dependência física, dificuldades para realização de atividades de vida diária e declínio $\operatorname{cog}_{\text {itivo }}{ }^{15}$. Pois, as consequências decorrentes da exposição à violência geram traumas e adoecimentos. Estudos demonstram que esses danos podem gerar isolamento, transtornos psicológicos e emocionais, dependência para atividades, sentimentos de humilhação, vergonha e, até mesmo suicídio ${ }^{16}$. Infelizmente, essa temática ainda é considerada estigma na sociedade, sendo relevante refletir sobre ela.

Nesse contexto, o medo também está inserido, principalmente em situações de crise como na pandemia COVID-19, o receio é fortalecido pela insegurança, ausência de alternativas para tratamento ou vacina, diversidade de direcionamentos e informações, divulgação contínua de dados sobre mortalidade, além de dificuldades econômicas, sociais e relacionais. O medo da morte é de extrema significância no contexto pandêmico. Porém, ele não se faz presente apenas na velhice, perpassa o processo de desenvolvimento humano e está presente no cotidiano da Pandemia, podendo gerar estresse ${ }^{17}$.

Nesse sentido, em revisão integrativa sobre o enfrentamento da morte, identificou-se que as situações estressantes podem ser provenientes de demandas internas ou externas. A primeira refere-se a situações relacionadas com o limite da capacidade e de autoconceito, como o próprio envelhecimento e morte; e, a outra refere-se a eventos traumáticos ou que apresentam ameaça ao bem-estar do indivíduo, como esse momento de pandemia. As estratégias de enfrentamento dependem das experiências vividas pelos idoso podendo ser: favoráveis, como a aceitação, conforto espiritual, procura por suporte social; desfavoráveis, como luto antecipado e isolamento ${ }^{18}$.

No que tange à procura por suporte social, a família é a principal provedora de cuidado e apoio. Assim sendo, deve-se considerar que seus membros também podem experienciar sentimentos de medo, preocupação, ansiedade e sobrecarga mediante ao cuidado de seu ente querido. Em arranjos familiares que coabita o idoso, requer-se atenção redobrada, com implementação de medidas preventivas da COVID-19. Ademais, a coabitação e convivência contínua pode gerar conflitos relacionais, causando aumento da tensão nesse período de pandemia, como vimos, a violência.

O distanciamento social também estimula alteração da rotina por parte dos membros familiares, para fornecer apoio ao idoso, como aquisição de alimentos, medicamentos ou outras necessidades. Em algumas famílias, além da ausência do contato físico com o idoso, também houve redução dos vínculos sociais, principalmente devido às dificuldades de acesso às tecnologias de informação (celulares e internet), incluindo a inabilidade para manuseá-las. 
Diante dos diversos desafios postos pela pandemia da COVID-19, é necessário vislumbrar estratégias de enfrentamento e possibilidades para ações junto aos idosos saudáveis e familiares. Nesse ínterim, a enfermagem gerontológica tem função essencial e primordial.

\section{POSSIBILIDADES DE AÇÕES PARA IDOSOS SAUDÁVEIS: CONTRIBUIÇÃO DA ENFERMAGEM GERONTOLÓGICA}

O distanciamento social envolve ações individuais e coletivas, que remodelam comportamentos, com prioridade para ações de higiene constantes como: higiene das mãos, uso de álcool em gel, distanciamento de outras pessoas, etiqueta respiratória, uso de máscaras, distanciamento de aglomerações, cuidados ambientais e emocionais.

Contudo, é preocupante a não adesão da população as medidas propostas. Além disso, há necessidade de atenção e cuidado aos comportamentos que envolvem os idosos, a rede de apoio e os familiares com contato junto a população de risco. Tendo em vista que a falta de adesão pode estimular a disseminação do SARS-CoV-2, prejudicando outras pessoas, além de sobrecarregar o sistema de saúde.

Estar em distanciamento social não significa que o idoso precisa permanecer no domicílio sem atividades. Muitas ações podem e devem ser realizadas para benefícios físicos, emocionais e sociais, com vistas a melhorar a interação e possibilitar as ações de lazer. Elencam-se como possibilidades ações que primariamente respeitem as preferências do idoso, incluindo: alongamento, caminhada, dança, leitura, escrita, contato telefônico com outras pessoas, realização de passatempos, prática de jogos variados, criação de algo novo, desenho, costura, restauração de objetos, bordado, prática de instrumentos musicais, cantar, ouvir música, de preferência dentro do espaço da casa, conversar com outras pessoas mantendo a distância necessária e evitando visitas domiciliares, rever fotos antigas, ter contato com animais domésticos, cozinhar, rezar, meditar, orar, além de cuidados com a casa e com materiais são relevantes.

A pandemia COVID-19 instigou maior reconhecimento pelo poder público das necessidades de conhecimento científico, profissionais capacitados, importância da pesquisa científica e desenvolvimento de outras formas de assistência em saúde, como o telecuidado. O recolhimento das pessoas em suas casas tem gerado novos hábitos na população, como os sistemas de telemonitoramento em saúde e a utilização de redes de apoio para aqueles integrantes dos grupos de risco.

Algumas experiências têm ocorrido com intuito de diminuir o impacto emocional do distanciamento social. Cita-se o Projeto Escuta Solidária que foi idealizado por docente na Universidade Federal de Viçosa e tem se expandido para outras universidades como: Federal do Tocantins, Estadual de Campinas e Federal do Estado do Rio de Janeiro. Essa ação presta apoio social a idosos, com escuta qualificada por meio de ligações telefônicas. O grupo que conduz a ação em Palmas (Tocantins) também tem acolhido os cuidadores de idosos acamados, na perspectiva de auxiliar as famílias tanto em aspectos emocionais quanto de cuidado.

A Universidade Federal do Paraná (UFPR), por meio da campanha "Pergunte aos cientistas", também disponibilizou canal de atendimento, para sanar dúvidas da população, sendo desenvolvidas orientações para aquelas mais prevalentes, auxiliando a aproximação do conhecimento científico da sociedade e evitando fake news. Outra iniciativa relevante, envolvendo o Hospital de Clínicas e Departamento de Enfermagem da UFPR é a criação da função de enfermeiras de ligação, que atuam no planejamento de alta, educação em saúde e monitoramento pós alta na contrarreferência entre o ambiente hospitalar e atenção primária, promovendo a integralidade e continuidade do cuidado aos pacientes suspeitos ou confirmados com COVID-19.

Também iniciativas de solidariedade têm surgido em vários municípios. Dentre elas, citam-se os vizinhos solidários que são pessoas do mesmo condomínio, que se colocam à disposição dos idosos em distanciamento para auxiliar em compras de alimentos e medicamentos. Outra iniciativa foi a abertura de aulas online e bate-papo com dicas e atividades interativas além de uma programação online relacionada a saúde física e mental utilizando tecnologia e socialização para a pessoa idosa. 
No âmbito profissional, salienta-se as plataformas de educação a distância, com disponibilidade de diversos cursos online gratuitos sobre a temática COVID-19, propostos por Instituições de Ensino Públicas e privadas, assim como Governo Federal. Na enfermagem, a Universidade Federal de Santa Catarina (UFSC) em parceria com Conselho Federal de Enfermagem, promoverão oferta de 300 mil vagas para profissionais de enfermagem realizarem cursos de atualização com foco em biossegurança e assistência ao paciente crítico no contexto da COVID-19. Além disso, diversos periódicos científicos abriram chamadas específicas para a temática e publicaram artigos com diversas vertentes que envolvem o período pandêmico.

Também a Associação Brasileira de Enfermagem, por meio do Departamento Científico de Enfermagem Gerontológica, desenvolveu diretrizes, vídeos, orientações, documentos técnicos, e jogos sobre o assunto. Na mesma vertente, a Sociedade Brasileira de Geriatria e Gerontologia, disponibilizou lives sobre temáticas envolvendo idosos e COVID-19, além de documentos técnicos e criação de Comissão Especial de Enfrentamento da COVID-19.

Essas ações fortalecem a enfermagem gerontológica no contexto pandêmico, promovendo atuação relevante e direcionadora para as demandas emergentes. Os profissionais de enfermagem comprometidos para a promoção da saúde, investem esforços desenvolvendo capacitações, possibilitando atuação qualificada e atualizada, incluindo desenvolvimento de materiais criativos para orientações, interações, informações para os idosos e familiares durante o distanciamento social.

\section{CONSIDERAÇÕES FINAIS}

As adversidades impostas pela pandemia COVID-19 são diversas, incluindo crises no âmbito econômico, social, de saúde e relacional. Em tempo de pandemia o distanciamento social se faz necessário para proteger de idosos e demais grupos de risco, achatar a curva epidemiológica e, como consequência, evitar o colapso no setor saúde. Contudo, essa medida traz desafios e oportunidades.

As ações de distanciamento social incitaram principalmente nos idosos solidão, desamparo, angústias, medos e preocupações. Além disso, vivenciou-se diversos episódios que reforçaram o ageísmo, assim como a violência contra os idosos. Este momento, reforçou a necessidade da rede de apoio fortalecida do idoso, assim como compreensão sobre ações preventivas para evitar a disseminação do SARS-CoV-2.

Ao mesmo tempo que a pandemia trouxe conflitos, algumas mudanças reforçam possibilidades, como a compreensão de que o distanciamento social perpassa ocupar o tempo com medidas sanitárias, atividades físicas, evidencia a preciosidade do toque, afeto, abraço, estar junto, ser apoio. Assim como, a relevância do conhecimento científico, a necessidade de profissionais capacitados e preparados para a assistência de enfermagem gerontológica, ações pioneiras/inovadoras e co-responsabilidade envolvida no cuidado do idoso, entrelaçada pelo próprio indivíduo, sua família e profissionais.

As diversas ações exitosas desenvolvidas por instituições públicas e privadas em prol de melhorias durante a pandemia, reforçaram as possibilidades e potencialidades, principalmente para capacitação de profissionais, despertando e reforçando a relevância de compreender a heterogeneidade do idoso e a primordialidade da enfermagem gerontológica na atenção à saúde.

A enfermagem gerontológica, ao longo de sua história, esteve junto aos idosos nos diversos cenários e contextos do envelhecimento. Nesse momento ímpar não seria diferente. Juntos no propósito e separados pelo distanciamento, segue-se tecendo caminho de lutas, enfrentamentos, oportunidades e inovações. Não se sabe qual será desfecho dessa pandemia e do distanciamento social, mas acredita-se que as vivências promoverão reflexão, respeito e dignidade para os idosos.

\section{AGRADECIMENTO}

Ao Departamento Científico de Enfermagem Gerontológica da ABEn Nacional. 


\section{REFERÊNCIAS}

1. Ferreira MJ, Irigoyen MC, Consolim-Colombo F, Saraiva JFK, Angelis K. Vida Fisicamente Ativa como Medida de Enfrentamento ao COVID-19. Arq Bras Cardiol. [Internet] 2020 [cited 2020 abr 18]. Available from: http://www.scielo.br/ pdf/abc/2020nahead/0066-782X-abc-20200235.pdf

2. World Health Organization. Statement on the second meeting of the International Health Regulations (2005) Emergency Committee regarding the outbreak of novel coronavirus (2019-nCoV) [Internet]. Geneva:World Health Organization; 2020 [cited 2020 Mar 27]. Available from:https://www.who.int/news-room/detail/30-01-2020-statement-on-the-second-meetingof-theinternational-health-regulations-(2005)-emergency-committee-regarding-the-outbreak-of-novel-coronavirus-(2019nov)

3. Ministério da Saúde. Boletim 4. Infecção Humana pelo Novo Coronavírus (2019-nCoV). [Internet]. 2020. [acesso 2020 mar 31]. Disponível em: https://www.saude.gov.br/images/pdf/2020/fevereiro/04/Boletim-epidemiologico-SVS-04fev20.pdf

4. Ministério da Saúde (BR). Portaria MS/GM n. 188, de 3 de fevereiro de 2020. Declara Emergência em Saúde Pública de importância Nacional (ESPIN) em decorrência da Infecção Humana pelo novo Coronavírus (2019-nCoV) [Internet]. Diário Oficial da União, Brasília (DF), 2020 fev 4 [cited 2020 mar 27]; Seção 1:1. Available from: http://www.in.gov.br/web/dou/-/ portaria-n-188-de-3-de-fevereiro-de-2020-241408388

5. Garcia, LP, Duarte E. Nonpharmaceutical interventions for tackling the COVID-19 epidemic in Brazil. Epidemiol. Serv. Saúde, Brasília, 29(2): e2020222, 2020. Available from: http://www.scielo.br/pdf/ress/v29n2/2237-9622-ress-29-02-e2020222.pdf

6. Zhou F, Yu T, Du R, Fan G, Liu Y, Liu Z et al. Clinical course and risk factors for mortality of adult inpatients with COVID-19 in Wuhan, China: a retrospective cohort study. Lancet. 2020 [cited 2020 abr 20]; 395: 1054-62. Available from: https://www. thelancet.com/action/showPdf?pii=S0140-6736\%2820\%2930566-3

7. Veiga AMV. Imunidade e Envelhecimento. In: Freitas EV, Py L. Tratado de geriatria e gerontologia. Rio de Janeiro: Guanabara Koogan; 2017. p. 2116-2127.

8. Chen N, Zhou M, Dong X, Qu J, Gong F, Han Y et al. Epidemiological and clinical characteristics of 99 cases of 2019 novel coronavirus pneumonia in Wuhan, China: a descriptive study pdf icon. Lancet. [Internet]. 2020. [cited 2020 mar 31]. doi: https://doi.org/10.1016/ S0140-6736(20)30211-7

9. Organização Pan-Americana da Saúde [OPAS]. Folha informativa - COVID-19 (doença causada pelo novo coronavírus). [Internet] 2020. [cited 2020 mar 24]. Disponível em: https://www.paho.org/bra/index.php?option=com_content\&view=ar ticle\&id=6101: covid19\&Itemid=875.

10. Gorzoni ML. Envelhecimento Pulmonar. In: Freitas EV, Py L. Tratado de geriatria e gerontologia. Rio de Janeiro: Guanabara Koogan; 2017. p. 1568-1570.

11. Wang L, He W, Yu X, Hu D, Bao M, Liu H et al. Coronavirus disease 2019 in elderly patients: characteristics and prognostic factors based on 4-week follow-up. J Infect. [Internet]. 2020 [cited 2020 abr 22]; 11:20 doi: .https://doi.org/10.1016/j. jinf.2020.03.019.

12. Nunes VMAN, Machado FCA, Morais MM, Costa LA, Nascimento ICS, Nobre TTX et al. COVID-19 e o cuidado de idosos: recomendações para instituições de longa permanência. [Internet] Natal (RN): EDUFRN; 2020. [cited 02 abr. 2020]. Available from: https://repositorio.ufrn.br/jspui/handle/123456789/28754)

13. Barra RP, Moraes EN, Jardim AA, Oliveira KK, Bonati PCR, Issa AC et al. A importância da gestão correta da condição crônica na Atenção Primária à Saúde para o enfrentamento do Covid-19 em Uberlândia, Minas Gerais. APS em Revista. 2020; 2(1):38-43.

14. Carmona CF, Couto VVD, Scorsolini-Comin F. A experiência de solidão e a rede de apoio social de idosas. Psicol Estud. 2014; 19(4): 681-691.

15. World Health Organization. Elder abuse. 2018. [cited 2020 abr 18]. Available from: https://www.who.int/news-room/ fact-sheets/detail/elder-abuse

16. Hirsch, R. [Violência contra idosos]. Bundesgesundheitsbl. 2016; 59:105-112. https://doi.org/10.1007/s00103-015-2268-5 Alemão.

17. Farber SS. Envelhecimento e elaboração das perdas. Terc Idade Estud Envelhec [Internet]. 2012 [cited 2020 abr 18]; 23(53):7-17. Available from: https:// www.sescsp.org.br/files/edicao_revista/cd42b419-7df9-4182-8a57-4188279cf8a5.pdf.

18. Ribeiro MS, Borges NS, Araújo TCCF, Souza MCS. Estratégias de enfrentamento de idosos frente ao envelhecimento e à morte: revisão integrativa. Rev Bras Geriatr Gerontol. 2017; 20(6): 880-888. 